\title{
An Uncommon Severe Case of Pulmonary Hypertension - From Genetic Testing to Benefits of Home Anticoagulation Monitoring
}

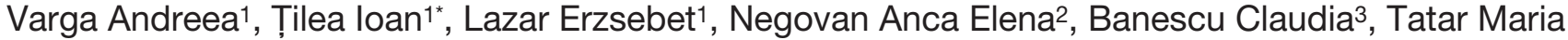 \\ Cristina2
}

1 Family Medicine, University of Medicine and Pharmacy Tirgu Mures, Romania

2 Internal Medicine III, University of Medicine and Pharmacy Tirgu Mures, Romania

${ }^{3}$ Genetics, University of Medicine and Pharmacy Tirgu Mures, Romania

\begin{abstract}
A 62 year-old caucasian male was admitted in our pulmonary hypertension expert center with initial diagnosis of pulmonary veno-occlusive disease for validation and specific treatment approach. Routine examinations revealed no apparent cause of pulmonary hypertension. Patient was referred for a thorax contrast enhanced multi-slice computed tomography which revealed extensive bilateral thrombi in pulmonary lower lobe arteries, pleading for chronic post embolic lesions. A right heart catheterization and pulmonary angiography confirmed the diagnosis of chronic thromboembolic pulmonary hypertension (CTEPH). Following the local regulations, the patient underwent thrombophilia screening including molecular genetic testing, with positive findings for heterozygous for VCORK1 -1639G>A gene single nucleotide polymorphism, PAl-1 4G/5G and factor II G20210A gene. With heterozygous genetic profile of 3 mutations he has a genetic predisposition for developing a thrombophilic disease which could be involved in the etiology of CTEPH. Familial screening was extended to descendants; the unique son was tested with positive results for the same three genes. Supportive pulmonary hypertension drug therapy was initiated together with patient selfmonitoring management of oral anticoagulation therapy. For optimal control of targeted anticoagulation due to a very high risk of thrombotic state the patient used a point-of-care device (CoaguChek®XS System, Roche Diagnostics) for coagulation self-monitoring.
\end{abstract}

Keywords: CTEPH, thrombophilia, gene polymorphism, heredity, anticoagulation self-monitoring management

\section{Background}

Chronic thromboembolic pulmonary hypertension $(\mathrm{CTEPH})$ is a distinct group (CTEPH and other pulmonary artery obstructions - WHO Group 4) among entities included in the large etiology of pulmonary hypertension, as they are defined in recent guidelines [1].

The diagnosis of CTEPH can be established after at least 3 months of anticoagulation therapy in patients with a mean pulmonary arterial pressure $\geq 25 \mathrm{mmHg}$ and pulmonary arterial wedge pressure $\leq 15 \mathrm{mmHg}$ associated with at least one segmental perfusion defect in V/Q lung scan and chronic organized occlusive thrombi in the pulmonary arteries revealed on CT pulmonary angiography [1].

Multiple conditions are associated with CTEPH: splenectomy, ventriculoatrial shunt, infected pace-maker, chronic inflammatory bowel disease, myeloproliferative disorders, antiphospholipid antibody syndrome, increased levels of factor VIII, malignancy, chronic venous ulcers, thyroid hormone replacement, non-O blood group $[2,3,4,5]$.

Prevalence and incidence CTEPH in real-life are unknown, it may occur in 5 individuals per million population per year [6] or up to $9.1 \%$ within the first 2 years after an acute episode of pulmonary embolism [7].

* Correspondence to: Ioan Tilea

E-mail: ioantilea2015@gmail.com
In well-selected cases pulmonary endarterectomy (PEA) is the treatment of choice, reducing pulmonary pressure and improving right ventricular function by removing chronic intravascular scar $[1,8,9,10]$.

In patients with no indication for surgery or with persistent/recurrent pulmonary hypertension after PEA, medical therapy $[1,11]$ or interventional procedures (percutaneous balloon angioplasty - PBA) are beneficial [12].

We describe a 62 year-old man heterozygous for VKORC1 G-1639A, PAI-1 4G/5G and factor II G20210A single nucleotide gene polymorphisms, and bilateral CTEPH after a unique thoracic pain episode with no hint for a thrombotic event. This case illustrates the importance of an extensive diagnosis in a PH/CTEPH expert centre and the severe clinical outcomes of the undiagnosed thrombophilic defects.

\section{Case report}

A white male aged 62, former smoker, was admitted in a county hospital in December 2014 with symptoms of coronary artery disease with a right bundle branch-block (RBB) on 12-leads rest ECG with no suggestive laboratory findings for an acute coronary syndrome; no other medical history of risk factors for cardiovascular disease were noticed. Due to concomitant symptoms and presence of RBB patient underwent TTE and native CT pulmonary angiography with results suggestive for pulmonary hyper- 
tension. Coronary angiogram was negative for atherosclerotic CAD.

Patient discharged diagnosis was severe pulmonary veno-occlusive disease (POVD group 1' WHO), congestive heart failure NYHA functional class III, chronic kidney disease stage II. Medical treatment was started. On discharge no oral anticoagulation regimen was started, at that moment there was no specific recommendation for anticoagulation therapy in PVOD patients following 2009 ESC/ERS Guidelines for the diagnosis and treatment of pulmonary hypertension.

Due to persistence of resting dyspnoea and signs of right heart failure, in March 2016 the patient was admitted to $\mathrm{PH} / \mathrm{CTEPH}$ expert centre for reassessment and specific supportive therapy.

Blood tests revealed a total bilirubin of $3.3 \mathrm{mg} / \mathrm{dl}$, GGT: $91 \mathrm{u} / \mathrm{l}$, eGFR: $41 \mathrm{ml} / \mathrm{min} / 1.73 \mathrm{~m}^{2}$ (MDRD formula) and $\mathrm{O}$ (zero) blood type, positive Rh group.

Specific blood tests and imagistic studies for associated medical conditions of $\mathrm{PH}$ were negative for congenital and/or left heart disease, deep venous thrombosis, thyroid disorders, lung disease and/or hypoxia, malignancy, portal hypertension, connective tissue disease, antiphospholipid antibody syndrome, inflammatory bowel disease, HIV, drugs, toxins and radiation induced PVOD.

Rest ECG showed sinus rhythm with a $75 \mathrm{bpm}$ heart rate and right bundle branch block associated with left posterior fascicular block (figure 1).

Transthoracic echocardiography pointed out enlarged right heart cavities, moderate/severe tricuspid regurgitation, mild pulmonary regurgitation and enlargement of pulmonary artery (figure 2, 3).
Contrast enhanced computed pulmonary tomography revealed dilated main pulmonary artery branches (left 26 $\mathrm{mm}$, right $27 \mathrm{~mm}$ ) and extensive adherent pulmonary thrombi in bilateral inferior lobar pulmonary arteries, suggestive for chronic post embolic lesions.

Ventilation/perfusion (V/Q) lung scan was not available.

Echocardiographic and right heart catheterization characteristics are summarized in Table I

The 6MWD test confirmed reduced exercise capacity, with $77 \%$ of predicted distance, decrease in peripheral $\mathrm{O}_{2}$ saturation from $92 \%$ to $87 \%$ at the end of test with Borg scale dyspnoea 2.

A final diagnosis of CTEPH was established by CT pulmonary angiogram, right heart catheterization and pulmonary angiography. At this point POVD was infirmed.

Due to no potential causes suggestive for CTEPH, a thrombophilic status related to a gene mutation or to gene polymorphism was suspected.

Fresh whole blood samples collected on EDTA were used for isolation of genomic DNA. Genotyping of the investigated polymorphisms was performed by using the Polymerase Chain Reaction - Restriction Fragment Length Polymorphism (PCR-RFLP) method.

Absence of gene mutation for factor V G1691A (Leiden), factor V H1299R (R2), factor XIII V34L has been determined. Extended genetic tests were performed for the patient and his unique direct male descendant. Both cases were heterozygous for the VKORC1 G1639A gene single nucleotide polymorphism, PAI-1 4G/5G, factor II G20210A gene and the clinical final judgement was that a genetic predisposition for developing a thrombophilic disease can be considered.

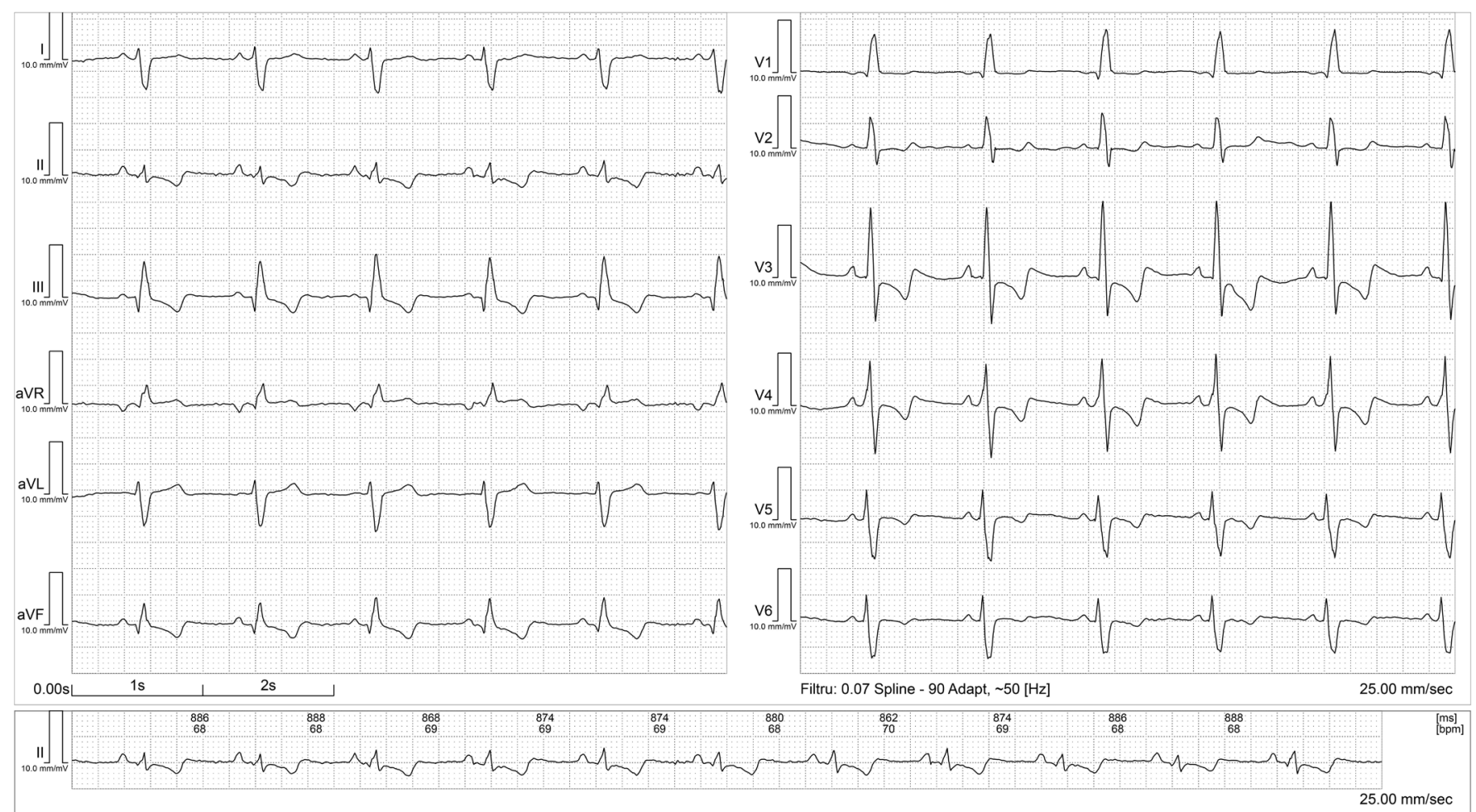

Fig. 1. Rest EKG -Sinus rhythm, right bundle branch block and left posterior fascicular block (RBBB+LPFB) 


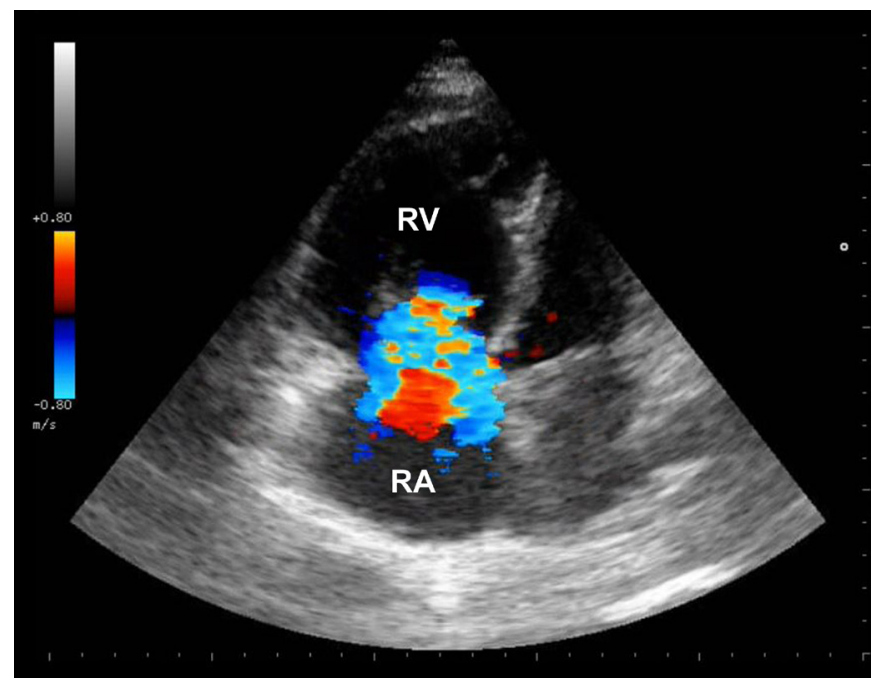

Fig. 2. Transthoracic examination. Moderate/severe tricuspid regurgitation, enlarged right ventricle (RV: $54.8 \mathrm{~mm}$ ) and right atrium (RA: $57.2 \mathrm{~mm}$ )

Surgical procedure (PEA) was refused by the patient, so a conventional therapy (oral anticoagulation, loop diuretics and aldosterone antagonists, oxygen supplementation) was started. Using a point-of-care device (CoaguCheck Roche Diagnostics, USA) the anticoagulation regimen with Acenocumarol was regularly adjusted to maintain the INR in therapeutic range. Specific therapy to decrease the pulmonary vascular resistance with endothelin receptor antagonist - Bosentan was initiated with a dose of $125 \mathrm{mg}$ b.i.d. Guanylate cyclase stimulator (Riociguat - class I level of evidence B, ESC 2015 guidelines indication) was not available at that time. Subsequent clinical follow-up visit was scheduled 4 weeks after hospital discharge for complete non-invasive medical evaluation in order to assess clinical and haemodynamic status and to adjust the therapy.

Table 1. Echocardiographic and right heart catheterization data

\begin{tabular}{lc}
\hline Echocardiography & \\
\hline LVEDD (mm) & 45 \\
IVS diastole (mm) & 9 \\
LAD (mm) & 35 \\
RVD (mm) & 46 \\
RA (mm) & 57 \\
LVEF (\%) & 55 \\
TAPSE (mm) & 29 \\
LV diastolic function & Abnormal relaxation \\
RV/RA pressure gradient (mmHg) & 100 \\
IVC diameter (mm) & 120 \\
PA pressure (mmHg) & 35 \\
Main PA diameter (mm) & NO \\
Pericardial effusion (mm) & NO \\
Pleural effusion & \\
Right heart catheterization & $53(89 / 36)$ \\
Mean pulmonary artery pressure & \\
(mPAP) - mmHg & 11 \\
Pulmonary capillary wedge pressure & \\
(PWP) - mmHg & $6.24(12.67)$ \\
Pulmonary vascular resistance (PVR) - & \\
HRU (HRUl) & $54 \%$ \\
O2 saturation pulmonary artery & $86 \%$ \\
O2 saturation aorta & \\
\hline
\end{tabular}

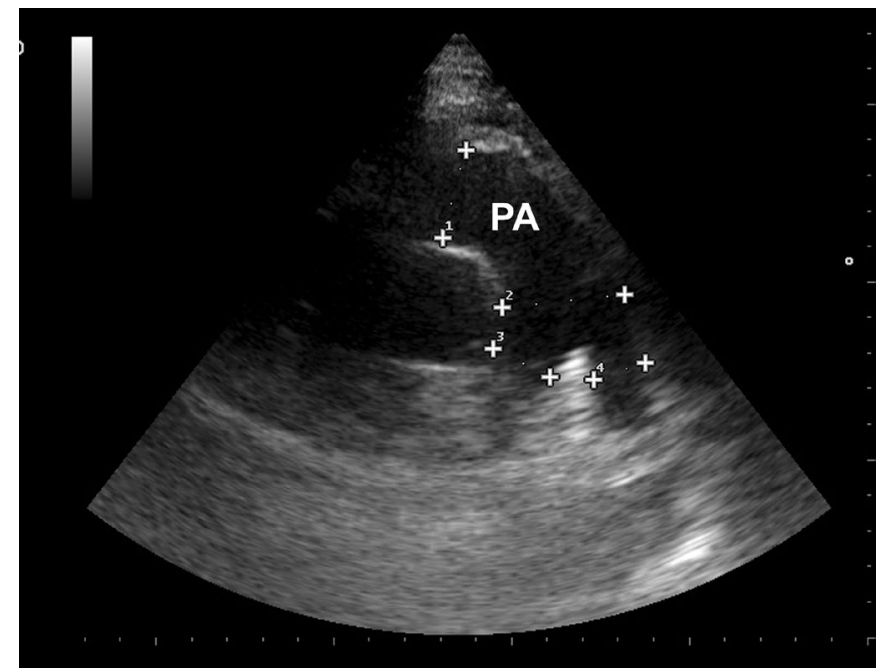

Fig, 3. Transthoracic examination. Enlarged main pulmonary artery and it's branches.

\section{Discussions}

Even if previously was thought that CTEPH is a rare cause of $\mathrm{PH}$, in expert centers precise imaging techniques and specific laboratory testing refines the cause of diagnosed cases of CTEPH as pulmonary embolism in $0,1-$ $9 \%$ cases [1].

In the absence of planar V/Q lung scan, modern CT pulmonary angiography can be an accurate method for the detection of CTEPH as it is suggested in recent works (96.1\% sensitivity, $95.2 \%$ specificity and $95 \%$ accuracy) $[12,13,14]$.

Although no specific genetic mutation have been linked to the development of CTEPH due to increased risk on genetic alteration, in cases with unclear etiology patients should be genetically screened for thrombophilic disorders after excluding the antiphospholipid and anticardiolipin antibody, lupus anticoagulant etiology.

The genetic predisposing factor is an important component in the mechanism of CTEPH patients. Li et al (2014) in a meta-analyse of 10 clinical case-control studies (7329 patients with cardiovascular disease and 7951 healthy controls) demonstrated that VKORC1 rs2359612 and rs9923231 polymorphisms correlate with high risks of cardiovascular and cerebrovascular diseases [15].

A meta-analyse performed by Wang J et al (2014) which included 34 studies with 3561 cases and 5693 controls suggested that PAI-1 $4 \mathrm{G} / 5 \mathrm{G}$ polymorphism is a risk factor venous thromboembolism [16].

Treatment algorithm of CTEPH brings to the forefront the recommendation of life long anticoagulation for all patients. (class IC recommendation) [1].

Beyond specific therapy with sGC stimulators in patients with CTEPH, anticoagulation and INR maintaining in therapeutic range plays a pivotal role for long term management and preventing recurrent thrombotic events in cases linked to thrombophilic disorders [17]. 


\section{Conclusions}

In CTEPH cases without clear etiology of the thrombotic event, a hypercoagulable state must be suspected. Extensive genetic testing should be conducted.

Descendants screening for heterozygous state susceptible for thrombotic events helps at-risk individuals for correct evaluation performed in a timely manner.

The clinician can offer a patient - centered approach to self-manage of their disease for INR monitoring that is associated with health and quality of life benefits.

\section{Consent}

Written informed consent was obtained from the patient for publication of this case report and any accompanying images. A copy of the written consent is available for review.

\section{Acknowledgement}

This work was supported by an Internal Research Grant of University of Medicine and Pharmacy Tirgu Mures, Romania, in partnership with SC Cosamext SRL Tirgu Mures, Romania, research contract number 8688/22.07.2015

\section{Competing interests}

The authors declare that they have no competing interests.

\section{Abbreviations \\ 6MWD 6 minutes walking distance \\ CI Cardiac index \\ CO Cardiac output \\ CT Computed tomography}

CTEPH Chronic thromboembolic pulmonary hypertension

IVC Inferior vena cava

LA Left atrium

LPFB Left posterior fascicular block

LV Left ventricle

LVEDD Left ventricle end-diastolic diameter

LVPW Left ventricle posterior wall

PAI-1 Plasminogen activator inhibitor-1

PH Pulmonary hypertension

PVR Pulmonary vascular resistance

RA Right atrium

RV Right ventricle

TAPSE Tricuspid annular plane systolic excursion

VKORC-1 Vitamin K epOxide Reductase Complex subunit

V/Q scan Ventilation/Perfusion Scan

PEA Pulmonary endarterectomy

PBA Percutaneous balloon angioplasty

RBBB Right bundle branch block

EKG Electrocardiogram

TTE Trans thoracic echocardiography

CT Computed tomography

CAD Coronary artery disease
POVD Pulmonary veno-occlusive disease

WHO World Health Organisation

NYHA New York Heart Association

$\mathrm{PH} \quad$ Pulmonary hypertension

MDRD Modification of Diet in Renal Disease

EDTA Ethylenediaminetetraacetic acid

DNA Deoxyribonucleic acid

PCR-RFLPPolymerase Chain Reaction - Restriction Fragment Length Polymorphism

\section{Conflict of interest}

The authors declare that they have no conflict of interest regarding the publication of this case report.

\section{References}

1. Galiè N, Humbert M, Vachiery JL, et al. 2015 ESC/ERS Guidelines for the diagnosis and treatment of pulmonary hypertension: The Joint Task Force for the Diagnosis and Treatment of Pulmonary Hypertension of the European Society of Cardiology (ESC) and the European Respiratory Society (ERS) Endorsed by: Association for European Paediatric and Congenital Cardiology (AEPC), International Society for Heart and Lung Transplantation (ISHLT). Eur Heart J. 2015;67-119

2. Matthews DT, Hemnes AR. Current concepts in the pathogenesis of chronic thromboembolic pulmonary hypertension. Pulm Circ. 2016;6(2):145-54

3. Wolf M, Boyer-Neumann C, Parent F, et al. Thrombotic risk factors in pulmonary hypertension. Eur Respir J 2000;15: 395-9.

4. Bonderman D, Turecek PL, Jakowitsch J, et al. High prevalence of elevated clotting factor VIII in chronic thromboembolic pulmonary hypertension. Thromb Haemost 2003;90:372-6

5. Lang I, Kerr K. Risk Factors for Chronic Thromboembolic Pulmonary Hypertension. Proc Am Thorac Soc 2006;3(7):568-70

6. Pepke-Zabka J, Jansa P, Kim NH, et al. Chronic thromboembolic pulmonary hypertension: role of medical therapy. Eur Respir $J$ 2013;41:985-90

7. Lang IM, Pesavento R, Bonderman D, Yuan JX. Risk factors and basic mechanisms of chronic thromboembolic pulmonary hypertension: a current understanding. Eur Respir J 2013;41(2):462-8

8. Fedullo PF, Auger WR, Kerr KM, et al. Chronic thromboembolic pulmonary hypertension. N Engl J Med 2001;345(20):1465-72

9. Mayer E, Jenkins D, Lindner J, et al. Surgical management and outcome of patients with chronic thromboembolic pulmonary hypertension: results from an international prospective registry. J Thorac Cardiovasc Surg, 2011;141(3):702-10

10. Jamieson SW, Kapelanski DP, Sakakibara N, et al. Pulmonary endarterectomy: experience and lessons learned in 1,500 cases. Ann Thorac Surg 2003;76(5):1457-62

11. Ghofrani HA, D'Armini AM, Grimminger $F$, et al. Riociguat for the treatment of chronic thromboembolic pulmonary hypertension. N Engl J Med. 2013; 369(4): 319-29

12. Satoh $T$, Kataoka $M$, Inami $T$, et al. Endovascular treatment for chronic pulmonary hypertension: a focus on angioplasty for chronic thromboembolic pulmonary hypertension. Expert Rev Cardiovasc Ther. 2016;14(9):1089-94

13. He J, Fang W, Lv B, et al. Diagnosis of chronic thromboembolic pulmonary hypertension: comparison of ventilation/perfusion scanning and multidetector computed tomography pulmonary angiography with pulmonary angiography. Nucl Med Commun 2012;33:459-63

14. Lang IM, Plank C, Sadushi-Kolici R, et al. Imaging in pulmonary hypertension. JACC Cardiovasc Imaging 2010;3:1287-95

15. LiY, Zhu J, Ding JQ. VKORC1 rs2359612 and rs9923231 polymorphisms correlate with high risks of cardiovascular and cerebrovascular diseases. Genet Mol Res. 2015;14(4):14731-44.

16. Wang J, Wang $\mathrm{C}$, Chen $\mathrm{N}$, et al. Association between the plasminogen activator inhibitor-1 4G/5G polymorphism and risk of venous thromboembolism: a meta-analysis. Thromb Res. 2014 ;134(6):1241-8

17. Heneghan C, Ward A, Perera R. Self-monitoring of oral anticoagulation: systematic review and meta-analysis of individual patient data. Lancet 2012; 379: 322-34 